\title{
Two-Dimensional X-Ray Grating Interferometer
}

\author{
Irene Zanette, ${ }^{1, *}$ Timm Weitkamp, ${ }^{1,2, \dagger}$ Tilman Donath, ${ }^{3}$ Simon Rutishauser, ${ }^{3}$ and Christian David ${ }^{3}$ \\ ${ }^{1}$ European Synchrotron Radiation Facility, Grenoble, France \\ ${ }^{2}$ Synchrotron Soleil, Gif-sur-Yvette, France \\ ${ }^{3}$ Laboratory for Micro- and Nanotechnology, Paul Scherrer Institut, Villigen, Switzerland
}

(Received 15 July 2010; revised manuscript received 11 October 2010; published 7 December 2010)

\begin{abstract}
We report on the design and experimental realization of a 2D x-ray grating interferometer. We describe how this interferometer has been practically implemented, discuss its performance, and present multidirectional scattering (dark-field) maps and quantitative phase images that have been retrieved using this device.
\end{abstract}

DOI: 10.1103/PhysRevLett.105.248102

PACS numbers: 87.59.-e, 07.60.Ly, 07.85.Qe, 42.30.Rx

$\mathrm{X}$-ray grating interferometry is a method for hard $\mathrm{x}$-ray wave-front-sensing and phase-contrast imaging that has been developed over the past few years [1-5]. It is highly sensitive to subtle deviations of the local wave front propagation direction (differential phase contrast) and to scatter in the sample (dark-field contrast), can be used with large fields of view, and does not need a high degree of $x$-ray monochromaticity [4]. A grating interferometer implemented on a synchrotron source typically consists of two line gratings, used in an inline transmission geometry and placed one behind the other. The first grating, termed the "beam splitter," acts as a diffractive element. The second one is used as an absorption mask $[2,3]$ and is often referred to as the "analyzer" grating.

An interferometer of this kind, using specifically developed line gratings [6,7], gives contrast for wave front deviations or scattering in the direction perpendicular to the grating lines. However, structures in the sample that are oriented perpendicular to the lines are not visible. In addition, retrieval of the wave front phase not only requires exact knowledge of the boundary values, but also induces substantial artifacts due to the lack of information on the "blind" direction.

To overcome these limitations, grating-based imaging systems that use 2D structures, rather than line gratings, have already been proposed. Some of them are singlegrating setups, i.e., without an analyzer grating $[8,9]$. The grating used in these systems has a period large enough so that the intensity modulations it induces can be directly resolved by the detector. Other proposed systems [10,11] do have an analyzer element in front of the detector or incorporated with the detector itself. However, no experimental scattering images or integrated phase maps are reported in $[10,11]$.

In this Letter, we present the design and realization of a device, measurement strategy, and data analysis algorithm that yield high-quality reconstructed phase images and multidirectional dark-field images using a 2D interferometer that includes an analyzer grating.
The device presented here is conceptually similar to the 1D interferometer we have reported in the past [3] except that the gratings are structured in both transverse dimensions. Thus, the beam splitter is a $2 \mathrm{D}$ diffraction grating $(G 1)$; its active areas are preferably pure phase structures. The diffraction pattern created by $G 1$ is an array of intensity spots. The absorbing analyzer grating $G 2$ encodes the spot positions into intensity values on the detector (Fig. 1). The difference from a standard 1D grating interferometer is that diffraction on $G 1$ occurs not only along $x$ or $y$ but in both dimensions, and $G 2$ allows analysis of the signals in both dimensions.

In the 1D case (i.e., the case of line gratings), the variable parameters for a binary grating are only the material, structure thickness $t$, lateral period $p$, and duty cycle $\gamma=w / p$ (where $w$ is the line width). Usually a duty cycle of $\gamma=0.5$ is preferred for the beam splitter grating, to eliminate its nonzero even diffraction orders.

The design of a 2D grating, however, allows a variety of different unit cells. In this Letter we consider only the case of cells with fourfold symmetry, equal periods $p_{x}=p_{y}=$ $P$, and duty cycles $\gamma_{x}=\gamma_{y}=0.5$. Two of the simplest unit cells are what we shall henceforward refer to as "checkerboard" (CB-) and "mesh" (M-)type patterns;

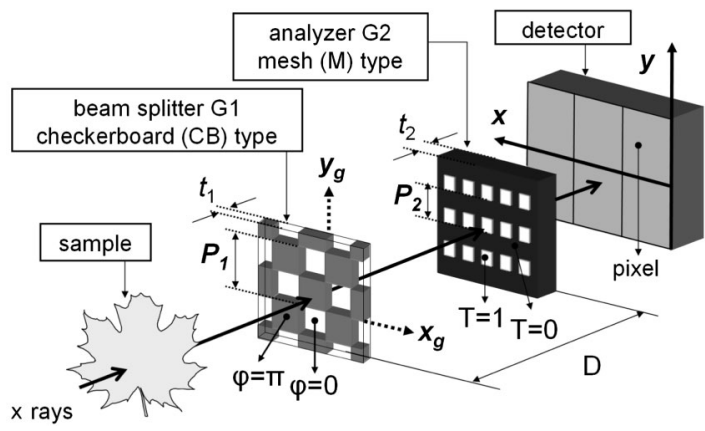

FIG. 1. Schematic representation of a $2 \mathrm{D}$ x-ray grating interferometer setup. The gratings $G 1$ and $G 2$ are placed in series in the X-ray path, separated by a distance $D . \varphi(x, y)$ denotes the phaseshift profile of $G 1, T(x, y)$ the intensity transmission of $G 2$. 
they are shown in Fig. 1. The intensity distribution produced by $G 1$, and the distances at which maximum contrast is observed, will depend on the pattern chosen and on the phase shift induced by the structures. A detailed study [12] shows that a CB-type, $\pi$-shifting grating produces a distribution of square intensity spots forming an $M$-type pattern with half the period of $G 1$. This phenomenon is observed at distances $D_{n}=n P_{1}^{2} /(8 \lambda)$, with $P_{1}$ the period of $G 1, n=1,3,5, \ldots$, and $\lambda$ the wavelength of the radiation. These distances may be different for other designs of a 2D diffraction grating [12].

The analyzer grating $G 2$ has opaque structures $[4,12]$. In our device, it is an $M$-type grating with period $P_{2}=P_{1} / 2$. As in the 1D case, $G 2$ has the same pattern as the (unperturbed) intensity distribution impinging on it.

The gratings forming the $2 \mathrm{D}$ grating interferometer can be implemented by using genuine 2D structures. However, for particular $G 1$ and $G 2$ patterns, it is also possible to combine two 1D gratings to create the equivalent of a 2D grating structure. A CB-type, $\pi$-shifting grating can be obtained as a superposition of two crossed linear $\pi$-shifting gratings; similarly, 2D mesh-type analyzer gratings can be obtained by the combination of two perpendicular linear absorption gratings [12].

The phase-stepping technique [4] can be used to separate absorption, phase, and scattering information. In the 2D case, a raster scan of one of the two gratings is performed along the transverse coordinates $x_{g}$ and $y_{g}$. The phase-stepping scan should be performed over at least one absorption grating period with at least three steps per period. The phase-stepping scan produces, in each detector pixel, a 2D intensity distribution which is the convolution of the interference pattern incident on this pixel with the transmission function of the analyzer grating. The simulated 2D convolution in the ideal case of fully coherent flat beam and perfect structures is shown in Fig. 2(a). The experimentally observed convolution resembles a $2 \mathrm{D}$ sinusoidal function [Fig. 2(b)].

For data analysis, it is useful to project the 2D signal recorded during a phase-stepping scan along four axes: the $x$ and $y$ coordinates and the diagonals $d 1$ and $d 2$ that form, respectively, $45^{\circ}$ and $135^{\circ}$ with the $x$ axis. The resulting contrast curves are shown in Figs. 2(c) and 2(d); the amplitude of each curve is related to the visibility of the intensity pattern along the respective direction. The visibility $V$, defined as $V=\left(I_{\max }-I_{\min }\right) /\left(I_{\max }+I_{\min }\right)$, where $I_{\max }$ and $I_{\min }$ are the maximum and minimum intensity values observed in the contrast curve, can be considered a figure of merit of the performances of the grating interferometer [4]. In an ideal 2D grating interferometer of the type presented here, the visibility along the main axes is $100 \%$, along the diagonals it is $36 \%$; see solid lines in Figs. 2(c) and 2(d). The limited spatial coherence of the $\mathrm{x}$-ray beam, grating imperfections, and limited absorption from $G 2$ structures reduce the visibility to measured values of $37 \%$ along $x$ and $y$ and $10 \%$ along the diagonals.

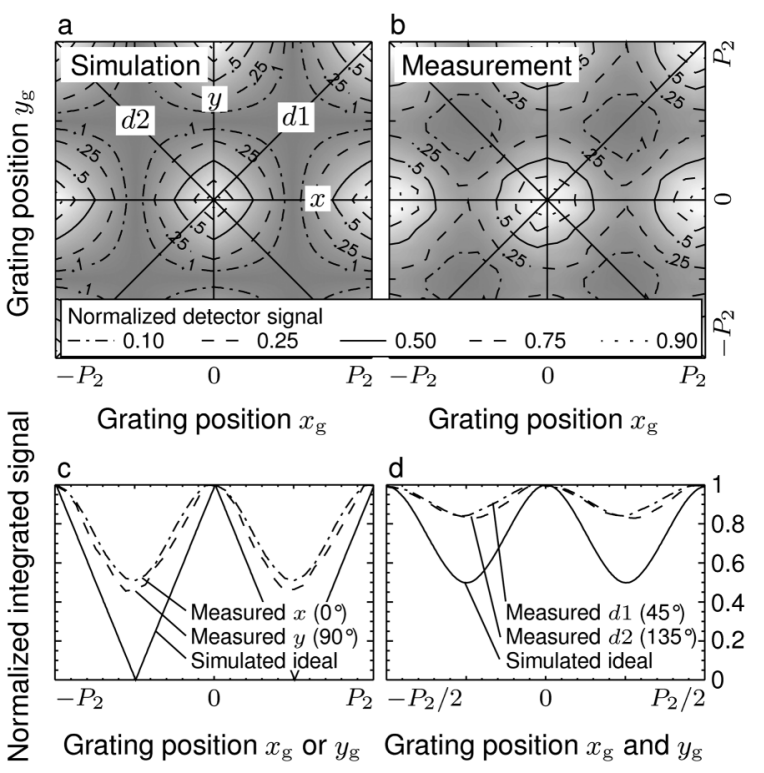

FIG. 2. Theoretical and experimental results of a 2D raster phase-stepping scan performed over one grating period. (The two-period representation used in this figure has been chosen for clarity. It is obtained by tiling the data). Panels (a) and (b) show the detector signal in a single pixel as a function of the grating coordinates $x_{g}$ and $y$. Panel (a) displays the calculated detector signal for ideal conditions (perfect gratings, fully coherent flat beam). Panel (b) shows the experimentally measured signal. The signal integrated along the $x$ and $y$ coordinates is shown in (c); the contrast curves along the diagonals $d 1$ and $d 2$ are shown in (d).

The measured visibility value of $37 \%$ along the main axes is comparable to the amplitude of the contrast curves obtained in the past with 1D grating interferometers [4,5]. The visibility value of $10 \%$ measured along the diagonals results in noisier images than along the main axes; however, as we will show in the following, scattering images along these directions give helpful information on the scattering orientation of the sample features.

The 2D intensity pattern in each pixel $(X, Y)$ can be expanded in a 2D Fourier series and, if the phase-stepping scan is performed over one period in each direction, it can be written as

$$
\begin{aligned}
I\left(X, Y, x_{g}, y_{g}\right) & =I\left(x_{g}, y_{g}\right) \\
& \simeq \sum_{k, l=-1}^{1} a_{k, l} \exp \left[\frac{2 \pi i}{P_{2}}\left(k x_{g}+l y_{g}\right)+i \phi_{k, l}\right],
\end{aligned}
$$

with the Fourier coefficients $a_{k, l}$ and their corresponding phases $\phi_{k, l}$ [note the analogy of Eq. (1) and the following treatment with the ones used for the 1D interferometer [5]]. From the Fourier analysis of the signal $I\left(x_{g}, y_{g}\right)$ in each pixel, the differential phase images, the scattering properties of the object, and the conventional absorption map can be simultaneously retrieved.

The differential phase shift along one transverse direction is related to the refraction angle in that direction by 
$\partial \Phi / \partial x=2 \pi \alpha_{x} / \lambda$ (analogous for $y$ ) [4]. The values of $\alpha_{x}$ and $\alpha_{y}$ can be calculated from the experimental data by

$$
\alpha_{x}=\frac{\phi_{0,1}^{\mathrm{sam}}-\phi_{0,1}^{\mathrm{ref}}}{2 \pi S}, \quad \alpha_{y}=\frac{\phi_{1,0}^{\mathrm{sam}}-\phi_{1,0}^{\mathrm{ref}}}{2 \pi S} .
$$

In the formulas above, $D$ is the distance between the two gratings and $S=D / P_{2}=2 D / P_{1}$ is the sensitivity of the interferometer [13]. The superscripts of Eq. (2) indicate values measured when the sample is in the beam path ("sam") and for reference phase-stepping scans ("ref").

Besides the two components $x$ and $y$ of the differential phase shift, a measure of the scattering power of the sample is obtained by calculating the decrease in visibility of the interference pattern caused by the sample. The scattering maps are usually called dark-field images [5]; we will indicate them with the letter $v$. With the 2D grating interferometer they can be retrieved along four directions [14]:

$$
\begin{aligned}
v_{x} & =A^{-1} a_{0,1}^{\mathrm{sam}} / a_{0,1}^{\mathrm{ref}}, & v_{y} & =A^{-1} a_{1,0}^{\mathrm{sam}} / a_{1,0}^{\mathrm{ref}}, \\
v_{d 1} & =A^{-1} a_{-1,1}^{\mathrm{sam}} / a_{-1,1}^{\mathrm{ref}}, & v_{d 2} & =A^{-1} a_{1,1}^{\mathrm{sam}} / a_{1,1}^{\mathrm{ref}} .
\end{aligned}
$$

The term $A^{-1}$ corrects for the absorption of the sample: $A=a_{0,0}^{\mathrm{sam}} / a_{0,0}^{\mathrm{ref}}$ is the absorption signal.

The experimental data presented here were taken with a setup mounted at the beam line ID19 [15] of the European Synchrotron Radiation Facility. The first grating was placed $150 \mathrm{~m}$ from the wiggler source. An x-ray energy of $23 \mathrm{keV}$ was selected by a Si-111 double crystal Bragg monochromator. The detector was a lens-coupled CCD system with a magnification of 1.87 and an effective pixel size of $7.5 \mu \mathrm{m}$, using a camera with $2048 \times 2048$ pixels and a powder scintillator.

We built the interferometer by using two orthogonal linear phase gratings and two orthogonal linear absorption gratings. The phase gratings $G 1$ with a period of $P_{1}=$ $4.785 \mu \mathrm{m}$ and a $\mathrm{Si}$ structure thickness of $29 \mu \mathrm{m}$ were made at the Paul Scherrer Institut [6]. The two analyzer gratings $G 2$ with a period of $P_{2}=2.4 \mu \mathrm{m}$ and a $\mathrm{Au}$ structure thickness of $50 \mu \mathrm{m}$ were fabricated at the Karlsruhe Institute of Technology [7]. The grating periods were matched in order to compensate for beam divergence at the 9th fractional Talbot order $(D=479 \mathrm{~mm})$. The sample was placed immediately upstream of the phase grating pair, the detector as close as possible to the absorption grating pair.

Raster phase-stepping scans were performed over one period and in 4 steps in each direction (16 frames in total) with an exposure time of $1 \mathrm{~s}$ per frame.

The two components $\alpha_{x}$ and $\alpha_{y}$ of the refraction angle for a leaf are shown in Figs. 3(a) and 3(b). The phase wrapping phenomenon shows up as a noiselike artifact in areas with a strong $\mathrm{x}$-ray phase gradient; this effect is visible in the petiole and in the veins of the leaf. Together with unknown boundary values, and/or lowfrequency errors due to statistical propagation of errors

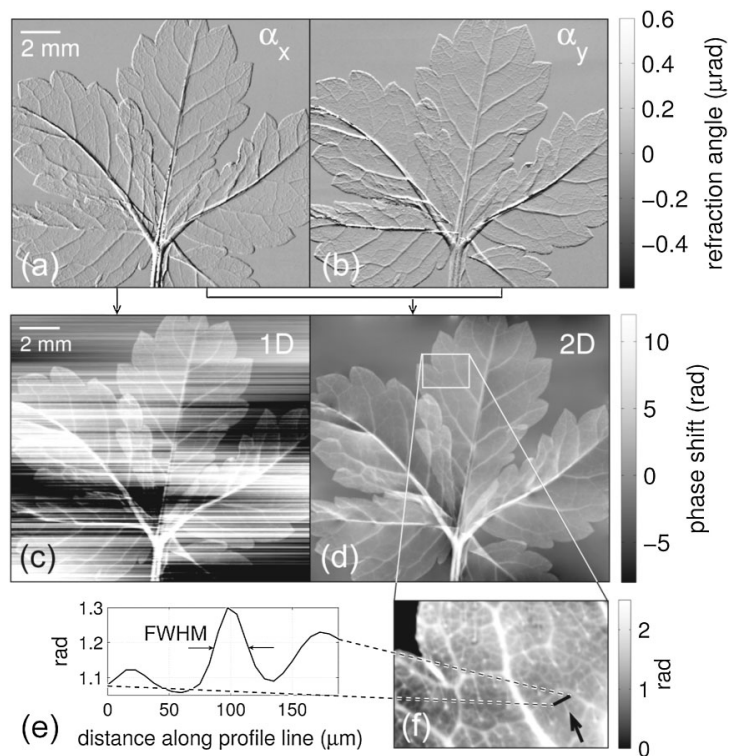

FIG. 3. Horizontal (a) and vertical (b) components of the refraction angle signal of a leaf recorded with a 2D grating interferometer. Note the superior quality of the $2 \mathrm{D}$ phase map retrieved with the $2 \mathrm{D}$ interferometer [(d) and detail (f)] over the one that would have been obtained with a 1D interferometer giving signal only along $x$ (c). The section profile across a linear feature in the phase map [plot in (e), profile position indicated by an arrow in (f)] has a FWHM of 3.9 pixels $(30 \mu \mathrm{m})$.

induced by noise, this phenomenon causes the phase map obtained with a 1D interferometer to contain strong stripe artifacts [Fig. 3(c)]. These artifacts are so strong that the phase projection maps are usually unusable as end results and therefore not shown in many publications on 1D interferometers. A substantial advantage of the 2D grating interferometer is the possibility of retrieving quantitative phase maps without these artifacts [Figs. 3(d) and 3(f)]. To obtain the phase map shown in Fig. 3(d) we adopted the Frankot-Chellappa method [16], widely used in optical microscopy. From this map, a lower limit of the spatial resolution in the phase image has been estimated by analyzing a section profile [Fig. 3(e)] across a hair on the leaf surface [the smallest structure discernible in the phase image indicated by an arrow in Fig. 3(f)]. The FWHM of the curve generated by the hair has been taken as a measure of the spatial resolution; it is $30 \mu \mathrm{m}$.

Figure 4 shows the central region of interest of the darkfield images of the leaf. The information in the diagonal directions helps to determine with more accuracy the preferential scattering direction of the sample features (e.g., details indicated by rectangles and circles in Fig. 4). Since the dark-field images obtained with the grating interferometer are sensitive to scattering features smaller than the detector pixel size, the multidirectional scattering images from a $2 \mathrm{D} x$-ray grating interferometer can be used to determine the orientation of structures in the sample with characteristic sizes well beyond the spatial resolution of the device. 

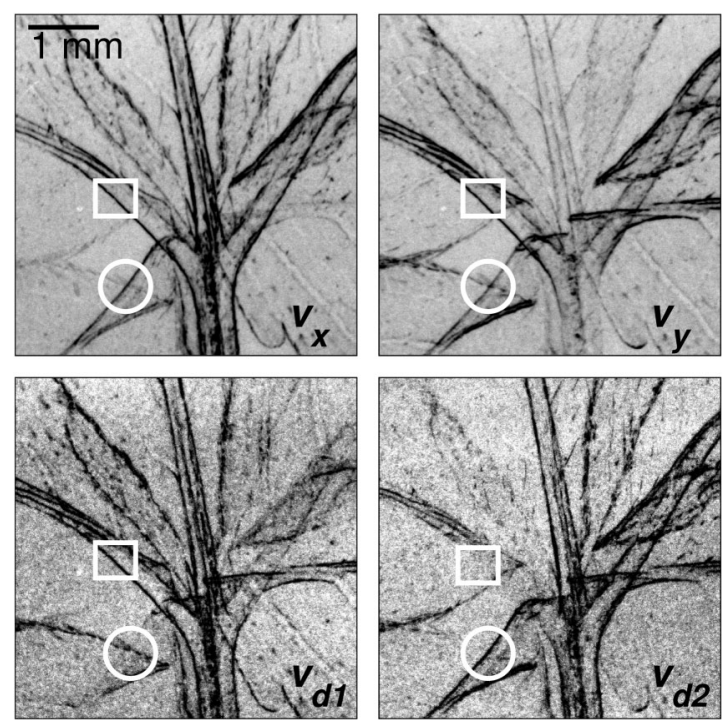

FIG. 4. Scattering (dark-field) images of the central part of the leaf along the four directions $(x, y, d 1$, and $d 2)$ as indicated in the bottom right of each image. Strong scattering details in the object are represented by dark regions in the images. Images $v_{x}$ and $v_{y}$ have a better signal-to-noise ratio than $v_{d 1}$ and $v_{d 2}$ because the visibility is higher along the main axes than along the diagonals (Fig. 2). Details highlighted with circles and squares are examples of features for which the diagonal information helps to determine with more accuracy their preferential scattering direction.

With the 2D interferometer presented here, it is possible to retrieve the phase and amplitude profiles of $x$-ray wave fronts and the scattering in the sample. The results presented show the substantial advantages of the 2D interferometer over a 1D design: higher quality of the retrieved phase images and directional scattering information in the dark-field signal.

Considerations concerning performance aspects of 1D grating interferometers (e.g., [4]), such as chromaticity, effects of imperfect spatial and temporal coherence, and spatial resolution, can also be applied to the $2 \mathrm{D}$ grating interferometer presented here.

Compared to other recently proposed schemes of $2 \mathrm{D}$ grating-based imaging [8,9], the effort of using a second grating is rewarded by higher sensitivity and spatial resolution. This is because, when an analyzer $G 2$ is used, the intensity modulation created by $G 1$ need not be resolved by the detector, and therefore gratings $G 1$ with period $P_{1}$ much smaller than the detector resolution can be used. Moreover, if the instrument is used in phase-stepping mode, the full spatial resolution of the imaging system is retained in the final images. This is not what happens in the single-grating setups, where the spatial resolution is limited by the period of the intensity pattern observed in the detector plane [9].

While we have demonstrated operation of the instrument in phase-stepping mode, it is also possible to use this device for moiré interferometry. In this mode, the phase profile can be retrieved from a single shot [3], albeit at inferior spatial resolution than in phase stepping. This mode may be interesting for various wave-front-sensing applications, particularly in the photon diagnostics for future $\mathrm{x}$-ray free-electron lasers.

The authors gratefully acknowledge Franz Pfeiffer, Martin Bech, and Martin Dierolf, Technische Universität München, Germany, for fruitful discussions, and Elena Reznikova and Jürgen Mohr, Karlsruhe Institute of Technology, Germany, for providing the absorption gratings used in these measurements.

*zanette@esrf.fr

†weitkamp@synchrotron-soleil.fr

[1] C. David, B. Nöhammer, H.H. Solak, and E. Ziegler, Appl. Phys. Lett. 81, 3287 (2002).

[2] A. Momose, Jpn. J. Appl. Phys. 42, L866 (2003).

[3] T. Weitkamp, B. Nöhammer, A. Diaz, C. David, and E. Ziegler, Appl. Phys. Lett. 86, 054101 (2005); see also [17]

[4] T. Weitkamp, A. Diaz, C. David, F. Pfeiffer, M. Stampanoni, P. Cloetens, and E. Ziegler, Opt. Express 13, 6296 (2005).

[5] F. Pfeiffer, M. Bech, O. Bunk, P. Kraft, E. F. Eikenberry, C. Brönnimann, C. Grünzweig, and C. David, Nature Mater. 7, 134 (2008).

[6] C. David, J. Bruder, T. Rohbeck, C. Grünzweig, C. Kottler, A. Diaz, O. Bunk, and F. Pfeiffer, Microelectron. Eng. 84, 1172 (2007).

[7] E. Reznikova, J. Mohr, M. Börner, V. Nazmov, and P. J. Jakobs, Microsyst. Technol. 14, 1683 (2008).

[8] K. L. Baker, Opt. Eng. (Bellingham, Wash.) 48, 086501 (2009), and references therein.

[9] H. H. Wen, E. E. Bennett, R. Kopace, A. F. Stein, and V. Pai, Opt. Lett. 35, 1932 (2010).

[10] A. Momose and S. Kawamoto, Jpn. J. Appl. Phys. 45, 314 (2006).

[11] A. Olivo, S.E. Bohndiek, J.A. Griffiths, A. Konstantinidis, and R.D. Speller, Appl. Phys. Lett. 94, 044108 (2009).

[12] I. Zanette, C. David, S. Rutishauser, and T. Weitkamp, AIP Conf. Proc. 1221, 73 (2010).

[13] T. Donath, M. Chabior, F. Pfeiffer, O. Bunk, E. Reznikova, J. Mohr, E. Hempel, S. Popescu, M. Hoheisel, M. Schuster, J. Baumann, and C. David, J. Appl. Phys. 106, 054703 (2009).

[14] Following the same principle, phase gradient images along the $d 1$ and $d 2$ directions can also been calculated. They are not presented here because the $x$ and $y$ components of the phase gradient are sufficient to retrieve the phase map.

[15] T. Weitkamp, I. Zanette, C. David, J. Baruchel, M. Bech, P. Bernard, H. Deyhle, T. Donath, J. Kenntner, S. Lang, J. Mohr, B. Müller, F. Pfeiffer, E. Reznikova, S. Rutishauser, G. Schulz, A. Tapfer, and J.P. Valade, Proc. SPIE Int. Soc. Opt. Eng. 7804, 780406 (2010).

[16] R. Frankot and R. Chellappa, IEEE Trans. Pattern Anal. Mach. Intell. 10, 439 (1988).

[17] T. Weitkamp, B. Nöhammer, A. Diaz, C. David, and E. Ziegler, Appl. Phys. Lett. 86, 119902 (2005). 